\title{
Adenovirus-mediated transfer of VEGF into marrow stromal cells combined with PLGA/TCP scaffold increases vascularization and promotes bone repair in vivo
}

Chunguang Duan ${ }^{1}$, Jian Liu' ${ }^{1}$, Zhi Yuann ${ }^{1}$, Guolin Meng ${ }^{1}$, Xiumei Yang ${ }^{2}$, Shuaijun Jia ${ }^{1}$, Jinkang Zhang' ${ }^{1}$, Shi Chen ${ }^{1}$

1Department of Orthopaedics and Traumatology, Xijing Hospital, Fourth Military Medical University, Xi'an, PR China

2Department of Ophthalmology, Xijing Hospital, Fourth Military Medical University, Xi'an, PR China

Submitted: 11 July 2011

Accepted: 23 December 2011

Arch Med Sci 2014; 10, 1: 174-181

DOI: $10.5114 /$ aoms.2012.30950

Copyright @ 2014 Termedia \& Banach

\section{Abstract}

Introduction: Large osseous defect remains a serious clinical problem due to the lack of sufficient blood supply and it has been proposed that this situation can be relieved by accelerating the formation of new vessels in the process of bone defect repair. The aim of this study was to develop a new type of artificial bone by transferring the VEGF gene into marrow stromal cells (MSCs) and seeding them into a porous scaffold.

Material and methods: An adenovirus vector was employed to transfer the VEGF gene into MSCs and expression of the exogenous gene was confirmed by ELISA. Next the transduced cells were seeded into a collagen I modified PLGA/TCP scaffold. The constructed new complex artificial bone was then assessed for biocompatibility in vitro and blood vessel formation and bone formation in vivo. Results: We found that adenovirus mediated VEGF gene transfer into MSCs sustained VEGF expression in MSCs for 3 weeks. Porous scaffold PLGA/TCP made by rapid prototyping technology exhibited improved biocompatibility resulting from crosslinking with collagen I. Furthermore, the in vivo study showed that large amounts of blood vessels were detected histologically 1 week after artificial bone implantation, and significant bone formation was detected 8 weeks after implantation.

Conclusions: Our findings suggest that gene transfer of VEGF into MSCs combined with PLGA/TCP scaffold enhances bone repair in vivo by promoting vascularization.

Key words: vascular endothelial growth factor, gene transfer, artificial bone, poly(DL-lactic-co-glycolic acid)/tricalcium phosphate.

\section{Introduction}

The clinical gold standard for osteogenic bone replacement in osseous defects is autologous bone grafting. When artificial bone is transplanted in vivo, its survival relies on interstitial fluid diffusion and blood perfusion. As the fluid diffusion can only support cells within a maximum range of $200 \mu \mathrm{m}[1,2]$, the survival rate of large cell-containing constructs will be limited by the lack of nutrition. Accordingly, the induction of vascularization is essential to the survival of the artificial bone in vivo.

\section{Corresponding author:}

Prof. Jian Liu

Department of Orthopaedics

and Traumatology

Xijing Hospital

Fourth Military

Medical University

No. 17 West Changle Road

710032 Xi'an, PR China

Phone: +862984771013

Fax: +86 2984771013 ,

E-mail:

duanchuanguang@hotmail.com 
Recent evidence indicated that a porous scaffold with suitable pore size could promote vascularization [3]. Poly(DL-lactic-co-glycolic acid)/tricalcium phosphate (PLGA/TCP) composite has emerged as a promising bone graft scaffold but its application is limited because it is not an effective hydrophilic material. To tackle this problem, many methods have been introduced [4], one of which is to crosslink PLGA/TCP with collagen I [5, 6]. Notably, the crosslink of PLGA/TCP and collagen I could accelerate bone repair [7].

Marrow stromal cells (MSCs) are ideal candidates for therapeutic applications $[8,9]$. The MSCs can be easily osteogenic induced by adding mineralization solution and have been applied in skeletal tissue repair $[10,11]$. Therefore, MSCs are suitable seeding cells in the attempt of constructing new complex artificial bone which can accelerate the process of both bone formation and vascular formation. Vascular endothelial growth factor (VEGF) is the most widely used growth factor to accomplish therapeutic angiogenesis $[12,13]$. Gene transfer of VEGF has been shown to induce neovascularization and increase blood flow with restored vasomotion in collateral vessels $[13,14]$ as well as at the microvascular level [15].

Therefore, in this study we attempted to develop a new complex artificial bone that could accelerate both bone formation and vascular formation. We transferred the VEGF gene into MSCs and then loaded the transduced cells on a PLGA/TCP/collagen I scaffold. Our results showed that the new complex artificial bone promoted vascular and bone formation in vitro and in vivo.

\section{Material and methods}

\section{Materials}

Poly(DL-lactide-co-glycolide) (PLGA/TCP) (PLGA/ TCP ratio $60: 40$, L/G ratio $50: 50$, MW 40,000$75,000)$ was obtained from Tsinghua University, Beijing, China. Collagen I was purchased from Sigma Aldrich (St. Louis, MO, USA).

\section{Preparation of adenovirus}

Adenovirus encoding VEGF was established by using the Ad-Easy system (Stratagene, La Jolla, CA). Production and purification of the recombinant virus were performed as described in our previous report [16]. Briefly, the gene of human VEGF165 (Genbank no: AF486837) was subcloned into pAdTrack-CMV one by one to yield pAdtrack-CMV-VEGF. The resultant plasmid was con-transformed into Escherichia coli strain BJ5183 with the adenoviral backbone plasmid pAdEasy-1. The recombinant adenoviral plasmid was selected on a kanamycin plate and confirmed by sequencing. Lastly, the recombinant adenoviral plasmids were transfected into 293 cells where they were packaged into virus particles (Ad-VEGF). Timematched EGFP-positive cells infected with empty pAd-Easy1 vector served as controls (Ad-Easy1). Viral titres were estimated by optical density and standard plaque assay.

\section{Cell culture and transduction with adenovirus}

Isolation of rabbit MSCs was performed according to the standard protocol. In brief, rabbit (female, 12 weeks old, $2.5-3 \mathrm{~kg}$ ) bone marrow was washed with DMEM (Dulbecco's modified Eagle medium) and then seeded on a plate at a density of $5 \times$ $10^{6}$ cells $/ \mathrm{cm}^{2}$ with medium (DMEM, $10 \%$ fetal calf serum [FCS]) at $37^{\circ} \mathrm{C}, 5 \% \mathrm{CO}_{2}$ in a humidified atmosphere. The medium was changed $72 \mathrm{~h}$ later. About 1 week later, the cells were detached and reseeded at a density of approximately 2000 cells $/ \mathrm{cm}^{2}$ and cultivated. The third passage cells were differentiated into the osteogenic cell lineage by replacing the expansion medium with differentiation medium (DMEM, 10\% FCS, $0.1 \mu \mathrm{M}$ dexamethasone, $10 \mathrm{mM}$ $\beta$-glycerolphosphate, $50 \mu \mathrm{M}$ l-ascorbic acid-2phosphate). Next the differentiated MSCs were infected with adenovirus PAd-VEGF encoding the VEGF gene, when the adherent cells reached subconfluence. Cells were washed with phosphate buffered saline (PBS) three times and place at $37^{\circ} \mathrm{C}$ for $10 \mathrm{~min}$, and then infected with a multiplicity of infection (MOI) of 1-300 pfu/cell in $1 \mathrm{ml}$ of infection buffer at $37^{\circ} \mathrm{C}$ for $2 \mathrm{~h}$. After the infection, cell culture medium was added and the cells were cultured at $37^{\circ} \mathrm{C}, 5 \%$ $\mathrm{CO}_{2}$ in a humidified atmosphere. Fluorescence microscopic analysis of enhanced green fluorescent protein (EGFP) expression was carried out 3 days after the infection. At the same time, the concentration of VEGF was quantified in conditioned media of nontransduced or adenovirus transduced cells using a commercial ELISA kit (R\&D Systems Inc, Minneapolis) according to the manufacturer's instructions.

\section{Fabrication of VEGF modified porous PLGA/TCP/ collagen I scaffold}

PLGA/TCP copolymer scaffold was fabricated via rapid prototyping $[17,18]$. PLGA/TCP was crosslinked with collagen I according to the established method [5]. In brief, the sterilized scaffolds were shaped into $15 \mathrm{~mm} \times 5 \mathrm{~mm} \times 5 \mathrm{~mm}$ pieces, and then transferred into 24-well plastic culture plates. After being prewetted with culture medium overnight, $1 \times 10^{4}$ transduced MSCs (in $100 \mu \mathrm{l}$ medium) were seeded into each scaffold. Then $900 \mu$ l of culture medium was supplied $4 \mathrm{~h}$ later, and the culture was set at $37^{\circ} \mathrm{C}$ in $5 \% \mathrm{CO}_{2}$ humidified atmosphere. Scaffolds were divided into four groups:

- group A: infected MSCS +PLGA/TCP/collagen I;

- group B: MSCS+PLGA/TCP/collagen I; 
- group C: infected MSCs+PLGA/TCP;

- group D: MSCS+PLGA/TCP.

On day 3, 7 and 14, cell proliferation was detected by cell counting. In group A and C, cell culture medium was discard and $2 \%$ collagenase I was supplied. The scaffold was cultured in $2 \%$ collagenase at $37^{\circ} \mathrm{C}$ for $45 \mathrm{~min}$, and then cells were harvested and counted. In group B and D, the scaffold was cultured in $0.25 \%$ trypsin at $37^{\circ} \mathrm{C}$ for $5 \mathrm{~min}$, and then cells were harvested and counted. The VEGF secreted into culture medium was determined using a commercial ELISA kit at day 3, 6, 9, 12, 15 and 18.

\section{Scanning electron microscopy examination}

The scaffold was cut into $5 \mathrm{~mm} \times 5 \mathrm{~mm} \times 5 \mathrm{~mm}$ pieces. The cells were fixed on the scaffold after 2 days of culture with $2.5 \%$ glutaraldehyde in $0.1 \mathrm{~mol} / \mathrm{l}$ sodium cacodylate buffer $(\mathrm{pH}=7.3)$ for $3 \mathrm{~h}$ at room temperature, rinsed three times with PBS, and dehydrated in a grade ethanol series. Samples were then critically point-dried, coated with gold and observed by SEM (Hitachi X-650).

\section{In vivo study}

Forty-eight mature Newland rabbits (female, 12 weeks old, $2.5 \mathrm{~kg}$ ) were used according to guidelines approved by the Council of Animal Protection of the Fourth Military Medical University, Xi'an, China. Rabbits were divided into four groups: group A, the scaffolds with MSCs transduced by pAd-VEGF (12 rabbits, 24 radii); group $B$, the scaffolds with untransduced MSCs (12 rabbits, 24 radii); group $C$, the pure PLGA/TCP/collagen I scaffold (12 rabbits, 24 radii); group $D$, blank control, no scaffold was filled in defect (12 rabbits, 24 radii). The scaffold was established by the previous method. $1 \times 10^{8}$ transfected cells were seeded in the scaffold of group A, and $1 \times 10^{8}$ untransfected cells were seeded in the scaffold of group B. The animals were operated on under general anaesthesia. Surgery was performed under sterile conditions. The animal model was adapted from Wittbjer et al. as described previously [17-19]. Briefly, unilateral 15-mm critical-size defects were created in the distal radial diaphysis. A superomedial incision of $3 \mathrm{~cm}$ was made over the distal radius, soft tissues were dissected, and the bone was exposed by gentle retraction of the muscles. A 15-mm segmental diaphyseal defect was created with an oscillating saw under irrigation with $0.9 \%$ sterile saline solution. The periosteum was removed with the bone and $5 \mathrm{~mm}$ of periosteum was stripped from each side of the remaining proximal as well as distal main fragment. The defect was irrigated with sterile physiological saline solution, and the scaffolds were press fitted into the defect. Muscles, fascia and skin were separately closed over the defect with 4-0 resorbable sutures. The wound was closed in layers. Postoperatively, $4 \mathrm{mg} / \mathrm{kg}$ body weight of carprofen was given as needed for pain.

\section{Histological analysis}

Samples were harvested 1 and 8 weeks postoperatively. The sample size was 12 for each group at each time point. The samples were dehydrated in a graded series of alcohol (80-100\%) and embedded in polymethyl methacrylate (PMMA) without decalcification. Sections of $200 \mu \mathrm{m}$ thickness were cut using a microtome (SP1600, Leica, Germany). Then, the sections were polished to a thickness of about 40-50 $\mu \mathrm{m}$ using a special grinding machine (Exakt-Micro-Grindin System, Leica, Germany). Five sections of the central part of each sample were used for histomorphometric analysis. The Van Gieson stained sections were examined under light microscopy (DMLA, Leica). The area of the newly formed bone (NB) and the residual material (RM) were quantitatively evaluated using the Imagetool V3.0 software (UTHSCSA, USA). The NB (\%) and RM (\%) were calculated as follows:

$$
\begin{aligned}
& \text { NB }(\%)=\frac{\text { area of newly formed bone }}{\text { total area }} \times 100 \%, \\
& \text { RM }(\%)=\frac{\text { area of residual material }}{\text { total area }} \times 100 \% .
\end{aligned}
$$

\section{Immunohistochemistry staining}

The samples were embedded with polymethyl methacrylate and cut with a microtome (SP2500, Leica) into $8 \mu \mathrm{m}$ thin sections and stained for CD34 (Santa Cruz, California, USA).

\section{Micro-CT}

The samples were soaked in $10 \%$ neutro-formaldehyde for $48 \mathrm{~h}$, and then put into the micro-CT system (eXplore Locus SP Pre-Clinical Specimen MicroCT, GEHealthcare, USA). The $44 \mathrm{~mm}$ tube $21 \mu \mathrm{m} 150 \mathrm{~min}$ SS protocol standard was applied to scan samples to acquire continuous images, and bone formation was assessed after 3D reconstruction of acquired images.

\section{Statistical analysis}

Results were expressed as mean \pm standard deviation (SD). Statistical software SPSS 10.0 (Chicago, USA) was used to analyse the data by one-way ANOVA. All results were considered to be significant with $p<0.05$.

\section{Results}

\section{Adenovirus-mediated transfer of VEGF into marrow stromal cells}

The MSCs were successfully isolated from bone marrow and expanded up to passage three and differentiated into the osteogenic cell lineage which 

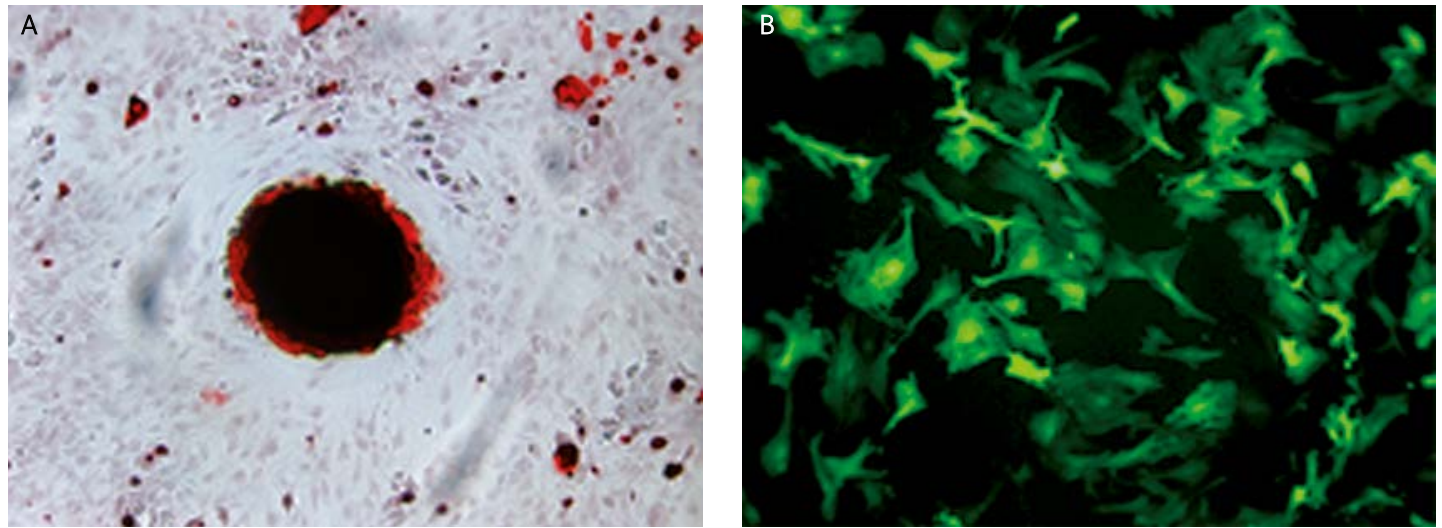

Figure 1. The MSCs differentiation and transduction with adenovirus. A - Alizarin Red S staining of osteogenic differentiated MSCs. B - adenovirus pAd-VEGF infected MSCs, MOI = 100

was identified by ALP and Alizarin Red S staining (Figure $1 \mathrm{~A}$ ). Thereafter, MSCs were infected by adenovirus pAd-VEGF. The transduction efficiency at MOI of 100 was about $85 \%$ by counting EGFP-positive cells (Figure $1 \mathrm{~B}$ ).

\section{Characterization of gene modified porous PLGA/TCP/collagen scaffolds}

The SEM analysis showed that the macro-pore dimension of PLGA/TCP scaffold was $500 \mathrm{~mm}$ in size with highly interconnective morphology (Figure 2). PLGA/TCP was crosslinked with collagen I. The cross-sectional morphology of the crosslinked scaffold showed porous microstructure with a high degree of interconnectivity (Figure 2). The magnified view of the pores was fairly uniform and roundshaped with a diameter of $300 \pm 50 \mu \mathrm{m}$ (Figure 2). The porosity was $85 \%$. Moreover, the differentiated MSCs were entrapped by the collagen I microsponges in the hybrid scaffold. After $8 \mathrm{~h}$ the cells adhered to the scaffold tightly and showed uniform distribution (Figure 2)

Water absorption of the PLGA/TCP scaffold was $3.06 \pm 0.67 \%$ while that of PLGA/TCP/collagen I was $12.42 \pm 1.45 \%(p=0.0022)$. The cell proliferation of all groups increased with culture time. On day 3 , the amount of cells in group A (infected MSCs+PLGA/ $\mathrm{TCP} /$ collagen I) was $38.75 \pm 0.9574$, in group $B$ (MSCs+PLGA/TCP/COL) $39.75 \pm 6.344$, in group C (infected MSCS+PLGA/TCP) $15.75 \pm 6.5$, and in group D (MSCs+PLGA/TCP) $19 \pm 2.793$. Significant differences exist between group $A$ and $C(p=0.004)$, and group $\mathrm{B}$ and $\mathrm{D}(p=0.0026)$. Between group $\mathrm{A}$ and $B$, and between group $C$ and $D$, the difference was not significant $(p=0.7658, p=0.4734$ respectively). On day 7 and 14 the amount of cells continued to increase while the relationship between groups was still the same as on day 3 (Figure 3). In other words, the same cells grew faster in the PLGA/TCP/collagen I scaffold than in the PLGA/TCP scaffold, and in the same scaffold, the growth speed of infected MSCs was not significantly different from the speed of non-infected MSCs.

The concentration of VEGF increased gradually within 9 days after infection and began to decrease afterwards. The Ad-VEGF infected MSCs seeded onto PLGA/TCP/collagen I scaffold (group A or group $B$ ) seemed to express more VEGF protein than those seeded onto PLGA/TCP scaffold (group C compared to group A, group D compared to group B), but the difference was not statistically significant (Table I).

\section{Improved vessel formation and bone formation after VEGF-modified artificial bone implantation}

Immunohistochemical staining of CD34 showed that one week after artificial bone implantation many new vessels appeared in VEGF transferred group but few new vessels could be observed in other groups (Figures $4 \mathrm{~A}-\mathrm{C}$ ). The vascular density was $85.76 \pm 13.87$ capillary $/ \mathrm{mm}^{2}$ in group $A, 24.00 \pm 11.40$ in group $B$, and 0 in group $C$. The vascular density of group $A$ was significantly higher than that of group $\mathrm{B}(p=0.0001)$ and $C(p<0.0001)$. Furthermore, 8 weeks after artificial bone implantation significant bone formation could be detected in the VEGF transferred group (group A), while in group $B$ (PLGA/TCP/Col scaffold with non-infected MSCs), bone formation could also be detected but the amount of new bone was less than in group $A$. In group $C$, little bone formation could be detected (Figures 4 D-F).

To further confirm whether VEGF transfer promoted bone formation, we performed micro-CT 8 weeks after transplantation and the results demonstrated that continuous regular new bone was formed in group $A$; the filling area accounted for $47.96 \pm 3.218 \%$ of the bone defect area. In group B, new bone was formed but was discontinuous. In addition, it only accounted for $18.42 \pm 1.608 \%$ of the bone defect area, significantly less than in group $A$ 
$(p<0.0001)$ In group C, a very small amount of new bone was found, only accounting for $2.260 \pm 1.626 \%$ of the bone defect area, significantly less than in group A $(p<0.0001)$, and group B $(p<0.0001)$ (Figures $4 \mathrm{G}-\mathrm{I})$. Taken together, these data suggest that VEGF-modified artificial bone implantation was able to significantly promote vessel and bone formation compared with normal artificial bone implantation.

\section{Discussion}

In this study we tried to accelerate the regeneration of bone through the establishment of VEGF modified artificial bone. In order to achieve this, we transferred VEGF into MSCs cells which were then seeded into a porous scaffold to construct artificial bone for implantation. Both in vitro and in vivo studies demonstrated that VEGF modified artificial bone promoted vascularization and bone formation.

The MSCs are promising in regeneration medicine especially in bone tissue engineering. Previous studies have proved that MSCs can be easily isolated from bone marrow and osteogenic differentiation of MSCs can be easily achieved by differentiation medium [20, 21]. Furthermore, MSCs have
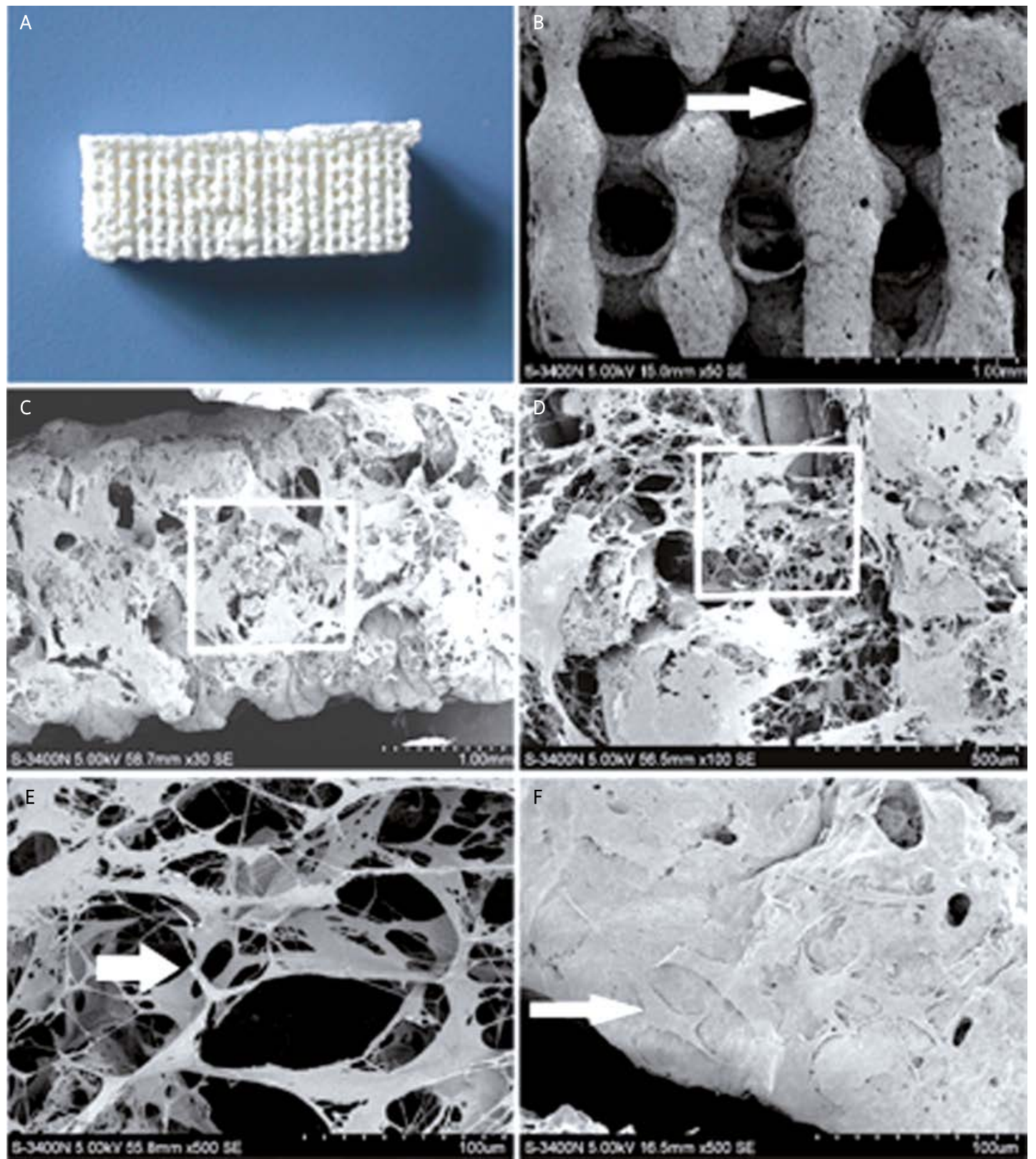

Figure 2. Morphological observations by SEM. A - PLGA/TCP scaffold. B - PLGA/TCP scaffold (50x), the arrows point to the PLGA/TCP frame. C - PLGA/TCP/collagen I scaffold (30x). D - Magnified view of the white rectangle frame from (C), demonstrating collagen I microsponge (100x). E - Magnified view of the white rectangle frame from (D), demonstrating collagen I microsponge (500x); the arrows point to the collagen I fibre. F-MSCs grown on hybrid scaffold (500x); the arrows point to the MSCs 
been demonstrated to successfully repair comparatively small osseous defects [19, 22, 23]. However, so far the application of MSCs to large bone defects repair has failed, mainly due to insufficient blood supply in the defected spots.

Therefore the main point is to address the insufficient blood supply in the process of repair of large bone defects. With the recent development of gene therapy, a very promising approach is to transfer proangiogenic factors such as VEGF to the defected area. Among the numerous gene transfer methods, we propose that adenovirus mediated gene transfer has an exceptional advantage in treatment of bone defects. First, the gene transferred by the adenovirus will not recombine with the host's genome. Therefore, this transfer method has relative high safety. Second, the crucial stage of bone regeneration is around 2-8 weeks, and adenovirus mediated gene transfer results in gene expression within 3-8 weeks. Thus the adenovirus gene transfer method is obviously able to complement the time course of the bone regeneration process. In contrast, other high efficient virus vectors such as retrovirus are not so safe and the expression is everlasting. Over-expression of the exogenous gene may do harm to the host [24, 25]. Therefore, adenovirus mediated gene transfer is a safe and efficient transfer method and is suitable for bone regeneration.

In order to achieve the aim of vascularization and osteogenesis, a suitable scaffold should have good biocompatibility and a suitable degradation rate. PLGA/TCP is a promising bone graft, but PLGA/ TCP is not effective hydrophilic material, and seeding cells cannot adhere to its surface easily. Collagen I plays a vital role in regulating cell adhesion and accelerating osteoblast proliferation [20-22, 26]. Therefore, collagen I crosslinked scaffold has the potential to accelerate the progress of bone repair. Indeed, Wu et al. reported that collagen I modified PLGA scaffolds increased the osteoblast attachment

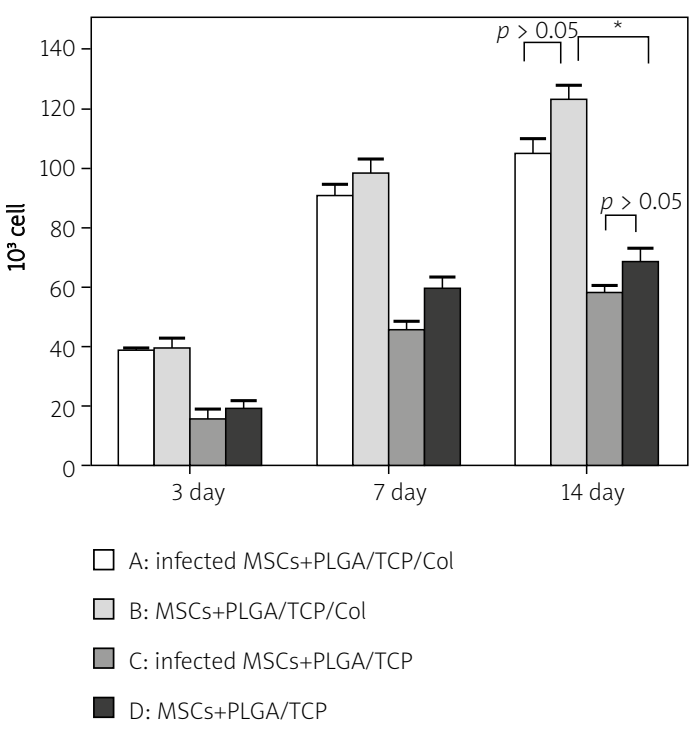

Figure 3. The proliferation of MSCs implanted in scaffolds. A - pAd-VEGF infected MSCs which were seeded onto PLGA/TCP/collagen I scaffold. B - non-infected MSCs which were seeded onto PLGA/TCP/collagen I scaffold. C - pAd-VEGF infected MSCs which were seeded onto PLGA/TCP scaffold. D - non-infected MSCs which were seeded onto PLGA/TCP scaffold $\left({ }^{*} p<0.05, n=4\right)$

and proliferation [23]. In this study, we also demonstrated that collagen I modified PLGA/TCP scaffold increased cell attachment and accelerated cell proliferation. Furthermore, we speculate that the new complex artificial bone constructed in this study promotes bone repair for the following two reasons, Firstly, the porous structure of the scaffold assisted the formation of new vessels while high expression of VEGF contributed to vascularization. Secondly, the microenvironment in the bone defect promotes osteogenesis. The MSCs can be differentiated into osteoblasts by local microenvironmental induction [27]. Interestingly, osteoprotegerin (OPG) was recently shown to be a promising agent for bone engineering

Table I. VEGF expression of established scaffold in vitro ( $\mathrm{pg} / \mathrm{ml}, \bar{x} \pm \mathrm{s}, n=4)$

\begin{tabular}{|c|c|c|c|c|c|c|c|}
\hline Group & 1 day & 3 day & 6 day & 9 day & 12 day & 15 day & 18 day \\
\hline $\begin{array}{l}\text { Group A } \\
\text { (infected } \\
\text { MSCs+PLGA/ } \\
\text { TCP/collagen) }\end{array}$ & $18.690 \pm 2.470$ & $81.05 \pm 5.453$ & $82.35 \pm 5.525$ & $147.60 \pm 13.86$ & $51.01 \pm 2.983$ & $21.98 \pm 2.290$ & $10.07 \pm 2.427$ \\
\hline $\begin{array}{l}\text { Group B } \\
\text { (MSCs+PLGA/ } \\
\text { TCP/collagen) }\end{array}$ & $0.263 \pm 0.098$ & $0.375 \pm 0.109$ & $0.420 \pm 0.098$ & $0.435 \pm 0.170$ & $0.373 \pm 0.113$ & $0.475 \pm 0.196$ & $0.438 \pm 0.209$ \\
\hline $\begin{array}{l}\text { Group C } \\
\text { (infected } \\
\text { MSCS+PLGA/ } \\
\text { TCP) }\end{array}$ & $17.78 \pm 2.121$ & $73.68 \pm 6.270$ & $77.23 \pm 4.848$ & $146.60 \pm 10.39$ & $44.62 \pm 4.638$ & $19.56 \pm 1.199$ & $9.343 \pm 2.716$ \\
\hline $\begin{array}{l}\text { Group D } \\
\text { (MSCs+PLGA/ } \\
\text { TCP) }\end{array}$ & $0.375 \pm 0.081$ & $0.393 \pm 0.101$ & $0.355 \pm 0.125$ & $0.310 \pm 0.095$ & $0.348 \pm 0.096$ & $0.373 \pm 0.140$ & $0.455 \pm 0.076$ \\
\hline
\end{tabular}



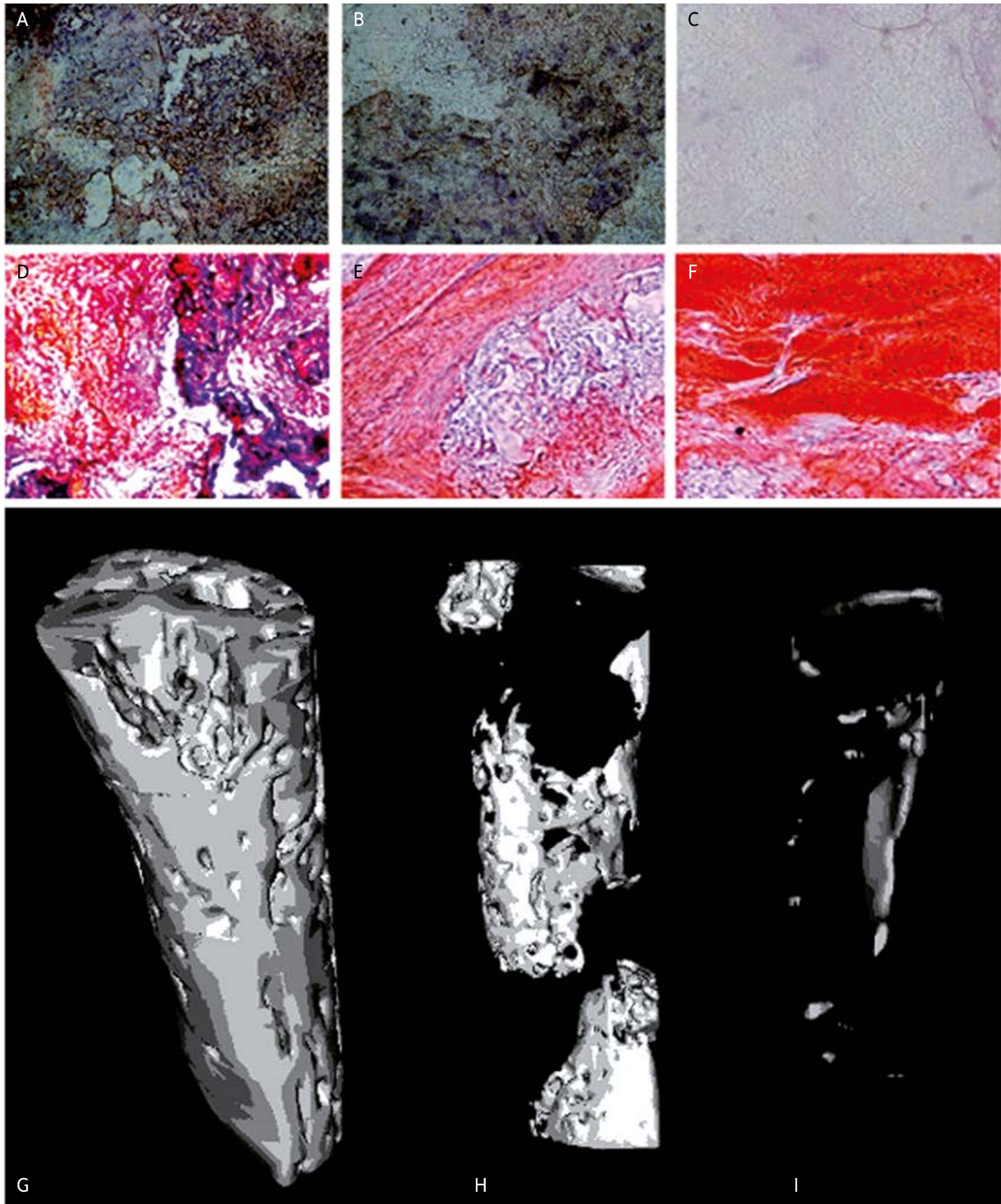

Figure 4. Improved vessel formation and bone formation after VEGF-modified artificial bone implantation. A-C - immunohistochemical staining of CD34 1 week after implantation. A - VEGF transferred group, B - PLGA/TCP/Col scaffold with non-infected MSCS, C - PLGA/TCP/Col scaffold. D-F - modified trichrome staining 8 weeks after implantation. D - VEGF transferred group, E - PLGA/TCP/Col scaffold with non-infected MSCs, F - PLGA/TCP/Col scaffold. G-I - MicroCT scan 8 weeks after implantation. G - VEGF transferred group, H - PLGA/TCP/Col scaffold with non-infected MSCs, I- PLGA/TCP/Col scaffold

$[28,29]$. Further investigation should aim at transfer of the OPG gene into MSCs to build OPG and VEGF double modified artificial bone for better therapeutic effects.

In conclusion, our data indicate that VEGF modified artificial bone facilitates large osseous defect repair in vivo, likely through enhanced vascularization. The combination of the adenovirus transfer system and collagen I modified PLGA/TCP scaffold represents a promising approach in bone tissue engineering.

\section{Acknowledgments}

Chunguang Duan, Zhi Yuan, Guolin Meng, Xiumei Yang and Shuaijun Jia contributed equally to this 
work. This work was supported by the National Natural Science Foundation of China (No. 30672142).

\section{References}

1. Goldstein AS, Juarez TM, Helmke CD, Gustin MC, Mikos AG. Effect of convection on osteoblastic cell growth and function in biodegradable polymer foam scaffolds. Biomaterials 2001; 22: 1279-88.

2. Folkman J, Hochberg M. Self-regulation of growth in three dimensions. J Exp Med 1973; 138: 745-53.

3. Klenke FM, Liu Y, Yuan H, Hunziker EB, Siebenrock KA, Hofstetter $\mathrm{W}$. Impact of pore size on the vascularization and osseointegration of ceramic bone substitutes in vivo. J Biomed Mater Res A 2008; 85: 777-86.

4. Pariente JL, Kim BS, Atala A. In vitro biocompatibility assessment of naturally derived and synthetic biomaterials using normal human urothelial cells. J Biomed Mater Res 2001; 55: 33-9.

5. Hao W, Hu Y, Wei $Y$, et al. Collagen I gel can facilitate homogenous bone formation of adipose-derived stem cells in PLGA-beta-TCP scaffold. Cells Tissues Organs 2008; 187: 89-102.

6. Jain RA. The manufacturing techniques of various drug loaded biodegradable poly(lactide-co-glycolide) (PLGA) devices. Biomaterials 2000; 21: 2475-90.

7. Hao W, Pang L, Jiang M, Lv R, Xiong Z, Hu Y. Skeletal repair in rabbits using a novel biomimetic composite based on adipose-derived stem cells encapsulated in collagen I gel with PLGA-beta-TCP scaffold. J Orthop Res 2010; 28: 252-7.

8. Borsani P, Marazzi R, Passi A, et al. Transplantation of bone marrow stromal mesenchymal cells in the treatment of acute myocardial infarction. Arch Med Sci 2009; 5: 156-62.

9. Liu X, Bao C, Hu J, Yin G, Luo E. Effects of clodronate combined with hydroxyapatite on multi-directional differentiation of mesenchymal stromal cells. Arch Med Sci 2010 6: 670-7.

10. Bianco P, Riminucci M, Gronthos S, Robey PG. Bone marrow stromal stem cells: nature, biology, and potential applications. Stem Cells 2001; 19: 180-92.

11. Xu J, Liu X, Chen J, et al. Cell-cell interaction promotes rat marrow stromal cell differentiation into endothelial cell via activation of TACE/TNF-alpha signaling. Cell Transplant 2010; 19: 43-53.

12. Takahashi $T$, Ueno $H$, Shibuya M. VEGF activates protein kinase C-dependent, but Ras-independent Raf-MEK-MAP kinase pathway for DNA synthesis in primary endothelial cells. Oncogene 1999; 18: 2221-30.

13. Gerber HP, McMurtrey A, Kowalski J, et al. Vascular endothelial growth factor regulates endothelial cell survival through the phosphatidylinositol 3'-kinase/Akt signal transduction pathway. Requirement for Flk-1/KDR activation. J Biol Chem 1998; 273: 30336-43.

14. Asahara T, Chen D, Tsurumi Y, et al. Accelerated restitution of endothelial integrity and endothelium-dependent function after phVEGF165 gene transfer. Circulation 1996; 94: 3291-302.

15. Takeshita S, Isshiki T, Ochiai M, et al. Endotheliumdependent relaxation of collateral microvessels after intramuscular gene transfer of vascular endothelial growth factor in a rat model of hindlimb ischemia. Circulation 1998; 98: 1261-3.

16. Duan CG, Meng GL, Liu J, et al. Construction of two-gene modified artificial bone by using recombinant adenovirus. Mater Sci Forum 2009; 610-613: 1343-55.

17. Wittbjer J, Palmer B, Thorngren KG. Osteogenetic properties of reimplanted decalcified and undecalcified autolo- gous bone in the rabbit radius. Scand J Plast Reconstr Surg 1982; 16: 239-44.

18. Geiger F, Bertram H, Berger I, et al. Vascular endothelial growth factor gene-activated matrix (VEGF165-GAM) enhances osteogenesis and angiogenesis in large segmental bone defects. J Bone Miner Res 2005; 20: 2028-35.

19. Kasten P, Vogel J, Geiger F, Niemeyer P, Luginbühl R, Szalay $\mathrm{K}$. The effect of platelet-rich plasma on healing in critical-size long-bone defects. Biomaterials 2008; 29: 3983-92.

20. Zambonin G, Grano M. Biomaterials in orthopaedic surgery: effects of different hydroxyapatites and demineralized bone matrix on proliferation rate and bone matrix synthesis by human osteoblasts. Biomaterials 1995; 16 : 397-402.

21. Caiazza S, Colangelo P, Bedini R, Formisano G, De Angelis $G$, Barrucci $S$. Evaluation of guided bone regeneration in rabbit femur using collagen membranes. Implant Dent 2000; 9: 219-25.

22. Brunel G, Brocard D, Duffort JF, et al. Bioabsorbable materials for guided bone regeneration prior to implant placement and 7-year follow-up: report of 14 cases. J Periodontol 2001; 72: 257-64.

23. Wu YC, Shaw SY, Lin HR, Lee TM, Yang CY. Bone tissue engineering evaluation based on rat calvaria stromal cells cultured on modified PLGA scaffolds. Biomaterials 2006; 27: 896-904.

24. Leppänen $P$, Kholová I, Mähönen AJ, et al. Short and longterm effects of hVEGF-A(165) in Cre-activated transgenic mice. PLoS One 2006; 1: e13.

25. Isner JM, Vale PR, Symes JF, Losordo DW. Assessment of risks associated with cardiovascular gene therapy in human subjects. Circ Res 2001; 89: 389-400.

26. Kleinman HK, Klebe RJ, Martin GR. Role of collagenous matrices in the adhesion and growth of cells. J Cell Biol 1981; 88: 473-85.

27. Gregory CA, Ylostalo J, Prockop DJ. Adult bone marrow stem/progenitor cells (MSCs) are preconditioned by microenvironmental "niches" in culture: a two-stage hypothesis for regulation of MSC fate. Sci STKE 2005; 2005: pe37.

28. Fili S, Karalaki M, Schaller B. Mechanism of bone metastasis: the role of osteoprotegerin and of the host-tissue microenvironment-related survival factors. Cancer Lett 2009; 283: 10-9.

29. Fili S, Karalaki M, Schaller B. Therapeutic implications of osteoprotegerin. Cancer Cell Int 2009; 9: 26. 